\title{
New Instruments and Saggestions
}

\section{AN ADJUSTABLE BRADFORD FRAME}

Robert D. Maddox, M.D., Cincinnati

A method of constructing Bradford and Whitman frames which overcomes some of the difficulties of preparation and use, without complicating the apparatus or greatly increasing the cost, has long been desirable. To fulfil these requirements, I have made a modification which, in dimensions and appearance, is essentially the accepted form of rectangular gaspipe frame for securing immobilization in recumbency. Each side of the frame is of one length of iron pipe, joined

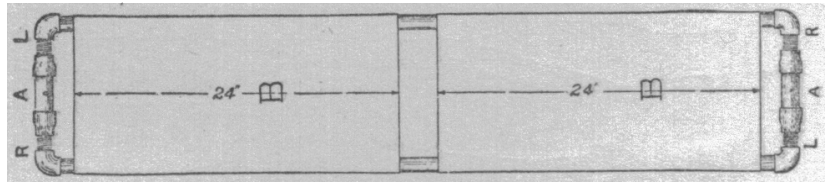

Fig. 1.-Adjustable Bradford frame.

at the ends by pieces made on the principle of the turnbuckle, so that the width can be varied. The canvas stretcher pieces are made in tubular form, and, when slipped over the ends of the frame, can be stretched tight by expanding the frame instead of the usual lacing (Fig. 1).

The side pieces are made in duplicate, of $1 / 2$ or $3 / 4$ inch pipe, 54 or 72 inches long, slightly bowed outward to draw the canvas tight in the center, and malleable iron elbows are fitted to each end. Fastened in these elbows, extending in the same plane but at right angles to the side pieces, are the threaded end pieces. These are of $1 / 2$ or $3 / 4$ inch pipe, 2 or 3 inches long and threaded the entire length, one right hand, and one left hand (Fig. 1, $R$ and $L$ ).

The turnbuckle sleeves are duplicates, from 4 to 14 inches long over all, and made of pipe sufficiently large to clear the threaded end pieces, with reducing fittings on each end, threaded right hand and left hand, to engage the threaded end pieces. A $3 / 8$ inch hole is drilled through the pipe in the center to hold the loose bar for turning (Fig. 1, $A, A$ ).

The canvas stretcher pieces are made of heavy cotton duck, with the selvage edge turned back for a reinforcing hem at the center end. The slides are overlapped and joined by a heavy seam, making a continuous band or tube of the canvas. When finished, the lengths of these pieces should be about $2 \frac{1}{2}$ inches less than half the length of the sides of

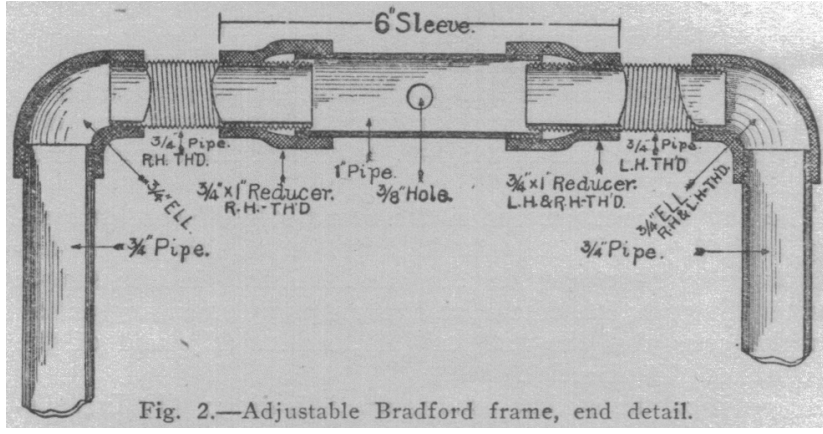

the frame, and the width from 8 to 18 inches, varying by half an inch up to 14 inches. They should be made in sets of four to permit changing, and the size should be plainly marked on each stretcher piece (Fig. 1, $B, B$ ).

To assemble the frame, the side pieces are laid parallel, an end piece threaded right hand opposite to an end piece threaded left hand, and the turnbuckle sleeves placed between them so that the right hand reducer will engage the right hand end piece, and the left hand reducer the left hand end piece at the same time. The sleeves are then turned equally and the sides are brought together so that the stretcher pieces can be slipped over each end. The sleeves are then turned in the opposite direction, forcing the sides apart and stretching the canvas.

As all parts of each size are interchangeable, frames of any width can be quickly assembled to meet the need of the individual patient by using sleeves of different lengths and stretcher pieces of proper width, a 4 inch sleeve expanding the 57 inch frame from 7 to 10 inches, and a 6 inch sleeve expanding the 75 inch frame from $91 / 2$ to $13 \frac{1}{2}$ inches, without the sleeves being changed.

The length of the frames is the maximum that can be placed on ordinary large or small hospital beds; but the fact that this length often exceeds the length of the patient by more than 4 inches does not appear to be of importance.

This type of frame has been used at the Episcopal Hospital for Children, Cincinnati, for more than a year, and it has been found that it can be prepared and the canvas changed and kept tight more easily than those of the usual pattern.

4 West Seventh Street.

\section{ASEPTIPHONE: AN ANTISEPTIC MOUTHPIECE FOR THE TELEPHONE}

E. M. Jenkins, M.D., Italy, Texas

The device shown in the accompanying illustration is designed to disinfect itself automatically and thereby minimize the danger of spreading $\mathrm{d}$ is e a se through this source. The instrument consists of several shells. The inner shell has a threaded portion for attaching to transmitter in place of the old mouthpiece, an annular flange extending laterally from the outer end of the inner shell so as to confine and embrace the outer shells. In the space between the outer and intermediate shells is a pad with disinfectant so-

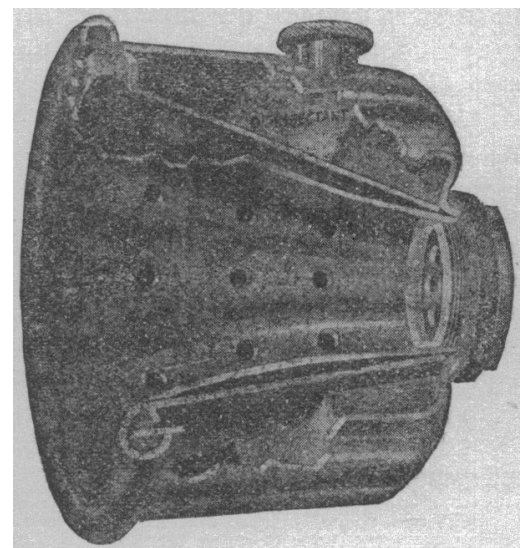

Antiseptic mouthpiece for telephone. lution. From this the fumes are disseminated, passing through an air chamber formed by the intermediate and inner shells, then out through the opening of the inner shells.

\section{A TRANSILLUMINATOR WITH NEW POSSIBILITIES}

William G. Reeder, M.D., Chicago

The transilluminator has become an important diagnostic agent in otorhinology. Through its use, pathologic changes in many of the accessory nasal cavities have become visualized. While the full significance of the picture it portrays may be difficult to interpret, it rarely deceives as to the fundamental facts desired-the presence or absence of disease. The instruments now in common usage require the electric current, and some a rheostat. They are expensive and of a size that does not permit them to be used within small cavities. I desire to call attention to an instrument which not only overcomes some of these objectionable features, but also, on account of its size and shape, has a far broader field of usefulness.

It consists of a small dry cell battery mounted in a case similar to an ordinary flashlight, which has a small but powerful lamp mounted at the free end of a rodlike projection and protected by removable caps, one fenestrated at the side, the other at the end.

To illuminate the antrum of Highmore, the lamp is carried upward between the lip anil the alveolar process until it rests 
firmly on the anterior surface of the superior maxillary bone at a point directly above the root of the second premolar tooth. The light rays now penetrate the thin anterior wall of the antrum, traverse the antral box, and emerge freely through the likewise thin floor of the orbit, illuminating, under normal conditions, the eliptic area bounded by the infra-orbital margin below and the eyeball above. The pupil of the eye will also show a red reflex in health. This position of the lamp has distinct advantages over the mouth position, as the rays of light pass through no tissue other than the walls of the antrum, thus eliminating the possibility of disease of other structures disturbing the picture. The walls traversed by the light when in this position are the thinner walls of the antrum, and they vary but little in thickness in different individuals, whereas all other walls may vary greatly, often making transillumination uncertain and indistinct. The presence of artificial denture does not interfere with this method.

The illumination of the frontal sinus is best accomplished by placing the lamp with the fenestrated cap well underneath the overhanging supra-orbital margin just external to the nasal process of the frontal bone and in contact with the roof of the orbit. This gives a brilliant and extensive illumination of the frontal sinus in health, so that the edges of the sinus in many cases may be perfectly outlined; indeed; often the location of partitions may be seen. Here, again, the light traverses the thin floor of the frontal sinus, and is not influenced by the varying thickness of the external table of the skull.

This instrument is a valuable mastoid illuminator. Its small size permits it to be passed into the osseous external auditory meatus of most persons, and by turning the illuminating window toward the posterior wall the mastoid cells are brilliantly illuminated. When the auditory meatus is small, the open end cap is used, allowing only the tip of the lamp to project beyond its free border in order that the meatus may be snugly. fitted, and preventing return rays of light from obscuring the picture. It may be used also by placing the lamp, with the rubber cap projecting slightly beyond it, against the external surface of the mastoid process and viewing the light that passes through the posterior osseous wall of the external auditory meatus.

The presence or absence of pathologic changes in the lacrimal sac may be elicited by this illuminator. Without the cap the lamp is passed into the nostril until it rests on the anterior end of the middle turbinate. In the presence of disease of the sac, a shadow is cast through the skin surface over the diseased area.

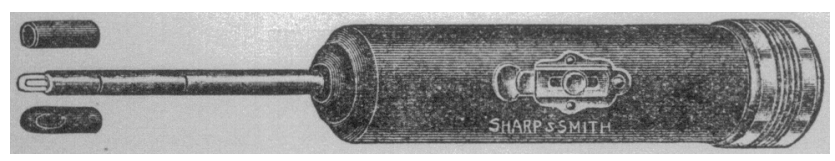

New transilluminator.

Likewise the lamp may be used in some cases to indicate disease of the anterior and middle ethmoidal cells, particularly in cases in which, with the added space secured by the use of cocain and epinephrin, the lamp may be passed between the middle turbinate and the septum. Disease of these structures is indicated by a shadow at the nasal portion of the orbit, and loss of the pupillary reflex.

This instrument is of value as a nasal diagnostic lamp. It is inserted into one nostril, and the interior of the nasal chambers of the other side is viewed through the vestibule opened by a nasal speculum. An unusual view of the interior of the nose may thus be obtained without the use of a head mirror.

This instrument is conveniently carried, and is ideal for bedside use and in homes where other means of illumination are not available. On account of its simplicity and its practicability, it is an instrument that will appeal to the general practitioner as well as the specialist.

Marshall Field Annex Building.

\section{NASAL SEPTUM . CLAMP OR SPLINT}

TO BE USED AFTER SUBMUCOUS RESECTIONS IN PLACE OF PACKING

\section{Frank C. Todd, M.D., Minneapolis}

The illustration represents a nasal septum clamp or splint which I have been using for the past year following submucous resection of the septum.

The clamp is placed in the nose immediately after the operation, and left in place for one or two days. No packing is used whatever, even though turbinates have been operated on at the same time. I find there is less bleeding than was

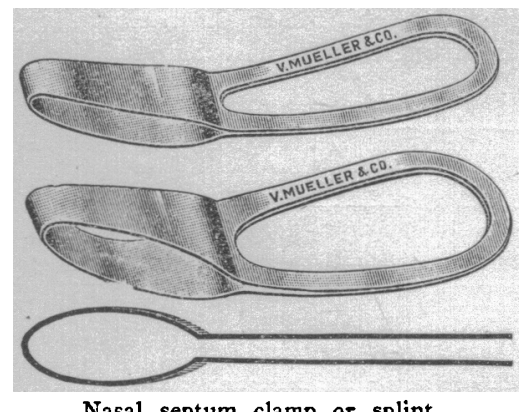

Nasal septum clamp or splint. the case when packing was used.

This device was shown before the Section on Laryngology, Otology and Rhinology at the San Francisco Session. It is made of German silver, so constructed that when inserted, with a wing on each side of the septum, a slight spring action of the two wings

holds the mucous membranes together. The clamp is much more comfortable than packing, and is more easily applied and removed.

My invariable custom, as was the case preceding the use of these clamps, is to keep the patient in the hospital at least two days after the operation.

These clamps come in two sizes, but the smaller size is rarely used. They are manufactured by V. Mueller \& Co.

\section{A SIMPLE METHOD OF QUANTITATIVE DETER- MINATION OF COMPLEMENT FIXATION}

\section{J. O. Hirschfelder, M.D., San Francisco}

In the complement fixation test the purpose is to determine the presence or absence in the serum to be tested of a body which on uniting with the antigen will prevent the complement from combining with the lysin to dissolve the blood cells.

In the antigen we have a complement fixing body $(A)$ and a substance $(B)$ which unites with the antibody in the serum to form a complement fixing substance. Similarly, in the serum we have a complement fixing body $(C)$ and the antibody $(D)$. The problem is to find the presence or absence of the antigenic substance and the specific antibody ( $B$ plus $D$ ) which on uniting fix the complement. If we wish to know how much of the latter combination is present, it is evident that we must first determine the amount of the anticomplementary action of the antigen and of the serum alone, as these vary.

The complement present in the serum of different guineapigs is highly variable, and, furthermore, rapidly diminishes on standing, even when kept in the ice chest. It is a simple matter, however, to determine the anticomplementary action simultaneously of the antigen $(A)$, serum $(C)$ and of the two mixed ( $B$ plus $D$ ).

In practice, the method used is the following: (1) Increasing quantities of complement; (2) increasing quantities of complement with a measured quantity of serum ; (3) increasing quantities of complement with a measured quantity of antigen, and (4) increasing quantities of complement with the quantities of serum and antigen used in 2 and 3 mixed are simultaneously incubated at $37 \mathrm{C}$. $(98.6 \mathrm{~F}$.) for one hour. To each of these tubes the same quantity of cells and lysin are then added, and all are incubated for two hours. At the end of that time they are read, and the least amount of complement necessary to dissolve the cells (termed the system) is determined.

The amount of complement necessary to dissolve the cells in presence of the serun minus the amount of the system 